VOL. 69 (2004) [289-295]

\title{
FINITE DIMENSIONAL CHARACTERISTICS RELATED TO SUPERREFLEXIVITY OF BANACH SPACES
}

\author{
M.I. OsTrovsKII
}

\begin{abstract}
One of the important problems of the local theory of Banach Spaces can be stated in the following way. We consider a condition on finite sets in normed spaces that makes sense for a finite set of any cardinality. Suppose that the condition is such that to each set satisfying it there corresponds a constant describing "how well" the set satisfies the condition.

The problem is: Suppose that a normed space $X$ has a set of large cardinality satisfying the condition with "poor" constant. Does there exist in $X$ a set of smaller cardinality satisfying the condition with a better constant?

In the paper this problem is studied for conditions associated with one of R.C. James's characterisations of superreflexivity.
\end{abstract}

\section{INTRODUCTION}

One of the most important and interesting classes of Banach spaces is the class of superreflexive Banach spaces. This class can be characterised in many different ways, see in particular $[2,4,7,10,11,12,22,24,25]$. See $[1,5,6]$ for exposition of some of characterisations of superreflexivity.

In this paper we are interested in the following characterisation that is due to James (see [1, pp. 236, 237, 265, 270] and [20, pp. 200-201]). By $B_{X}$ we denote the unit ball of a Banach space $X$.

THEOREM 1. The following three conditions are equivalent.

(i) The space $X$ is non-superreflexive.

(ii) For some $\delta>0$ and each $n \in \mathbf{N}$ there is a sequence $\left\{x_{i}\right\}_{i=1}^{n} \subset B_{X}$ such that

(1)

$$
\left\|\sum_{i=1}^{n} a_{i} x_{i}\right\| \geqslant \delta \cdot \sum_{i=1}^{n}\left|a_{i}\right|
$$

Received 23rd September, 2003

The author was partially supported by the Grant-in-aid of the Catholic University of America.

Copyright Clearance Centre, Inc. Serial-fee code: 0004-9727/04 \$A2.00+0.00. 
for each sequence $\left\{a_{i}\right\}_{i=1}^{n} \in \mathbf{R}^{n}$ with at most one change of signs.

(iii) For each $0<\delta<1$ and each $n \in \mathbf{N}$ there is a sequence $\left\{x_{i}\right\}_{i=1}^{n} \subset B_{X}$ such that (1) is satisfied for each sequence $\left\{a_{i}\right\}_{i=1}^{n} \in \mathbf{R}^{n}$ with at most one change of signs.

To discuss this result it is convenient to introduce the following notation. We denote the unit sphere of $l_{1}^{n}$ by $S_{1}^{n}$. We denote the subset of $S_{1}^{n}$ consisting of all sequences with at most one change of signs by $J_{n}$. (When we speak about changes of signs we ignore zero terms.)

Definition 1: Let $A \subset S_{1}^{n}$. For a Banach space $X$ we define the index $h(X, A)$ of $A$ in $X$ by

$$
h(X, A)=\sup _{\left\{x_{i}\right\}_{i=1}^{n} \subset B_{X}} \inf _{\left\{a_{i}\right\} \in A}\left\|\sum_{i=1}^{n} a_{i} x_{i}\right\| .
$$

With this definition Theorem 1 can be restated as

THEOREM 2. The following three conditions are equivalent.

(i) The space $X$ is non-superreflexive.

(ii) $\inf _{n} h\left(X, J_{n}\right)>0$.

(iii) $h\left(X, J_{n}\right)=1 \forall n \in \mathbf{N}$.

Using the standard ultraproduct argument (it is described, for example, in [1, p. 228]) it can be shown that Theorem 2 implies the following result.

TheOREM 3. For each $n \in \mathbb{N}$ and every real numbers $\alpha, \beta$ satisfying $1>\alpha$ $>\beta>0$ there is $N=N(n, \alpha, \beta) \in \mathbb{N}$ such that $h\left(X, J_{N}\right) \geqslant \beta$ implies $h\left(X, J_{n}\right) \geqslant \alpha$.

It is clear that Theorem 3 implies the equivalence (ii) $\Leftrightarrow$ (iii) from Theorem 2 .

In the local theory of Banach spaces it is important to have (as precise as possible) estimates for finite-dimensional parameters of Banach spaces. The main purpose of this paper is to estimate the function $N(n, \alpha, \beta)$ from below. It is worth mentioning that using the argument of [25] it is possible to estimate $N(n, \alpha, \beta)$ from above. The estimate obtained on these lines is a very rapidly increasing function, it is obtained by repeated use of the Ramsey theorem.

The main result of this paper states that for any pair $\alpha, \beta$ satisfying $1>\alpha>\beta$ $>0$ the value of $N(n, \alpha, \beta)$ considered as a function of $n$ grows more rapidly than any polynomial. We state this result in the following way.

THEOREM 4. Let $1>\alpha>\beta>0$. Then for each $m \in \mathbf{N}$ there is $n \in \mathbb{N}$ such that $h\left(X, J_{n^{m}}\right) \geqslant \beta$ does not imply $h\left(X, J_{n}\right) \geqslant \alpha$.

This result contrasts with the well-known lemma due to Giesy (see [8, Lemmas I.4 and I.6] or [16, p. 62]). Using the notation that we have introduced the lemma can be stated as

$$
h\left(X, S_{1}^{n^{2}}\right) \geqslant \alpha \Rightarrow h\left(X, S_{1}^{n}\right) \geqslant \sqrt{\alpha} .
$$


The estimate of $h\left(X, J_{n}\right)$ for a superreflexive space $X$ different from Euclidean seems to be a very difficult problem. For this reason our proof is indirect, it is based on some of the known results on the Kadets path distance. Let us recall necessary definitions.

Definition 2: Let $X$ be a Banach space and let $Y$ and $Z$ be closed subspaces of $X$. The opening (sometimes called also gap) between $Y$ and $Z$ is defined to be the Hausdorff distance between their unit balls, that is,

$$
\Lambda(Y, Z)=\max \left\{\sup _{y \in B_{Y}} \operatorname{dist}\left(y, B_{Z}\right), \sup _{z \in B_{Z}} \operatorname{dist}\left(z, B_{Y}\right)\right\} .
$$

If $X$ and $Y$ are arbitrary Banach spaces we define the Kadets distance

$$
d_{K}(X, Y)=\inf _{Z, U, V} \Lambda(U X, V Y)
$$

where the infimum is taken over all Banach spaces $Z$ and all linear isometric embeddings $U: X \rightarrow Z$ and $V: Y \rightarrow Z$.

Remarks. 1. The Kadets distance is a natural Banach-space analogue of the wellknown Gromov-Hausdorff distance between metric spaces. This analogue was introduced and studied by Kadets [13] in 1975 (before the publication of the famous paper Gromov [9]). See [15] and references therein for information on the Kadets distance.

2. Using standard techniques it can be shown (see [17, p. 110 of the English translation]) that $d_{K}$ satisfies the triangle inequality. It follows easily that the restriction of $d_{K}$ to the set of finite-dimensional Banach spaces is a metric. In the infinite-dimensional case $d_{K}$ does not separate points (there exist non-isomorphic $X$ and $Y$ such that $d_{K}(X, Y)=0$, see $[17$, Theorem 1c] or $[15$, p. 37]).

Definition 3: Let $X$ and $Y$ be Banach spaces. Suppose that there exists a continuous (with respect to the Kadets distance) mapping $Z:[0,1] \rightarrow$ B Banach spaces $\}$ such that $Z(0)=X$ and $Z(1)=Y$. We call such mapping a path joining $X$ and $Y$. The Kadets path distance $d_{K P}(X, Y)$ between $X$ and $Y$ is defined to be the infimum of the lengths with respect to the Kadets distance of all paths joining $X$ and $Y$.

If there is no path joining $X$ and $Y$, then $d_{K P}(X, Y)$ is defined to be $\infty$.

By the length of a path we mean the standard definition for metric geometry, see for example [3, p. 34]. The Kadets path distance was introduced and studied in [21].

Proof of THEOREM 4: The plan of the proof is the following.

1. We assume the contrary, that is, we assume that for some $0<\beta<\alpha<1$ and some $m \in \mathrm{N}$ the inequality $h\left(X, J_{n^{m}}\right) \geqslant \beta$ implies $h\left(X, J_{n}\right) \geqslant \alpha$ for every $n \in \mathbf{N}$.

2. We consider a sequence $\left\{X_{n}\right\}_{n=1}^{\infty}$ of spaces satisfying $h\left(X_{n}, J_{n}\right)=1$ and $d\left(X_{n}, l_{2}^{n}\right)=O(\ln n)$ (by $d(X, Y)$ we denote the Banach-Mazur distance, the existence of spaces $\left\{X_{n}\right\}$ satisfying the conditions was shown by Kadets [14], another example was found by Pisier and Xu [23]). 
3. We estimate the Kadets path distances $d_{K P}\left(X_{n}, l_{2}^{n}\right)$ from below and from above. The obtained estimates contradict each other.

To obtain an estimate for $d_{K P}\left(X_{n}, l_{2}^{n}\right)$ from above we use the following result from [21].

Theorem 5. Let $X$ and $Y$ be isomorphic Banach spaces. If $\ln d(X, Y) \geqslant \pi$, then

$$
d_{K P}(X, Y) \leqslant \pi \ln \ln d(X, Y) .
$$

Hence

$$
d_{K P}\left(X_{n}, l_{2}^{n}\right)=O(\ln \ln \ln n)
$$

To obtain an estimate from below we need the following (almost immediate) consequence of the triangle inequality for the norm of a Banach space (see [18] and [19, p. 292]).

Proposition 1. Let $X$ and $Y$ be Banach spaces, $A \subset S_{1}^{n}$ for some $n \in \mathbf{N}$. Then

$$
d_{K}(X, Y) \geqslant|h(X, A)-h(Y, A)|
$$

The inequality (3) can be used to find estimates for $d_{K P}(X, Y)$.

Proposition 2. Let $X$ and $Y$ be Banach spaces and let $A_{1}, \ldots, A_{k+1}$ be subsets of $S_{1}^{n}$ such that for some numbers $\alpha_{1}, \ldots, \alpha_{k}$ and $\beta_{1}, \ldots, \beta_{k}$ the following conditions are satisfied.

(A) $h\left(X, A_{j}\right) \geqslant \beta_{j}$ implies $h\left(X, A_{j+1}\right) \geqslant \alpha_{j}, j=1, \ldots, k$.

(B) $\alpha_{j}>\beta_{j+1}, j=1, \ldots, k$

(C) $h\left(X, A_{1}\right) \geqslant \beta_{1}$.

(D) $h\left(Y, A_{k+1}\right)<\alpha_{k}$.

Then

$$
d_{K P}(X, Y) \geqslant\left(h\left(X, A_{1}\right)-\beta_{1}\right)+\sum_{j=1}^{k}\left(\alpha_{j}-\beta_{j+1}\right)+\left(\alpha_{k}-h\left(Y, A_{k+1}\right)\right) .
$$

Observation. The conditions (A), (B), and (D) imply

(E) $h\left(Y, A_{j}\right)<\beta_{j} \forall j=1, \ldots, k$.

In fact, otherwise we would have $h\left(Y, A_{j}\right) \geqslant \beta_{j} \Rightarrow h\left(Y, A_{j+1}\right) \geqslant \alpha_{j}>\beta_{j+1}$ $\Rightarrow h\left(Y, A_{j+2}\right) \geqslant \alpha_{j+1}>\beta_{j+2} \Rightarrow \cdots \Rightarrow h\left(Y, A_{k+1}\right) \geqslant \alpha_{k}$, a contradiction.

Proof of Proposition 2. Let $X:[0,1] \rightarrow$ \{Banach spaces $\}$ be a path, continuous with respect to $d_{K}$, with $X(0)=X, X(1)=Y$.

We shall repeatedly use the following immediate consequence of $(3)$ : 
(F) For any fixed $A$ the index $h(X, A)$ is a continuous function of $X$ with respect to $d_{K}$.

By (F), (C), and (E) there exists $t_{1} \in[0,1]$, such that $0 \leqslant t_{1}<1$ and $h\left(X\left(t_{1}\right), A_{1}\right)$ $=\beta_{1}$. Hence $h\left(X\left(t_{1}\right), A_{2}\right) \geqslant \alpha_{1}$ (by (A)).

By (F), (B), and (E) there exists $t_{2} \in[0,1]$ such that $t_{1}<t_{2}<1$ and $h\left(X\left(t_{2}\right), A_{2}\right)$ $=\beta_{2}$. Hence $h\left(X\left(t_{2}\right), A_{3}\right) \geqslant \alpha_{2}$ (by (A)).

We continue in an obvious way. At the last step we find $t_{k}$ such that $t_{k-1}<t_{k}<1$ and $h\left(X\left(t_{k}\right), A_{k}\right)=\beta_{k}$. Hence $h\left(X\left(t_{k}\right), A_{k+1}\right) \geqslant \alpha_{k}$ (by (A)).

By the definition of $d_{K P}$ we get (by (3) used with different $A$ 's for different terms)

$$
\begin{gathered}
d_{K P}(X, Y) \geqslant d_{K}\left(X, X\left(t_{1}\right)\right)+\sum_{j=1}^{k-1} d_{K}\left(X\left(t_{j}\right), X\left(t_{j+1}\right)\right)+d_{K}\left(X\left(t_{k}\right), Y\right) \\
\geqslant\left(h\left(X, A_{1}\right)-h\left(X\left(t_{1}\right), A_{1}\right)\right)+\sum_{j=1}^{k-1}\left(h\left(X\left(t_{j}\right), A_{j+1}\right)-h\left(X\left(t_{j+1}\right), A_{j+1}\right)\right) \\
+\left(h\left(X\left(t_{k}\right), A_{k+1}\right)-h\left(Y, A_{k+1}\right)\right) \\
\geqslant\left(h\left(X, A_{1}\right)-\beta_{1}\right)+\sum_{j=1}^{k-1}\left(\alpha_{j}-\beta_{j+1}\right)+\left(\alpha_{k}-h\left(Y, A_{k+1}\right)\right),
\end{gathered}
$$

as was stated.

REMARK. Proposition 2 is a generalisation of a result from [21, Section 4].

We return to our proof of Theorem 4. Let $p \in \mathbb{N}$ be such that $h\left(l_{2}, J_{p}\right)<\beta$. Such $p$ exists because $l_{2}$ is superreflexive. To estimate $d_{K P}\left(X_{n}, l_{2}^{n}\right)$ from below we introduce the number

$$
s=\max \left\{i \in \mathbf{N}: p^{m^{i}} \leqslant n\right\} .
$$

It is easy to check that there exists an absolute constant $C>0$ such that

$$
s \geqslant C \cdot \ln \ln n
$$

for $n$ large enough. (We use the fact that both $p$ and $m$ are absolute constants in this argument.)

Let $r(i)=p^{m^{i}} i=0,1,2, \ldots, s$. We apply Proposition 2 with $k=s$,

$$
A_{1}=J_{r(s)}, A_{2}=J_{r(s-1)}, \ldots, A_{s+1}=J_{r(0)},
$$

$\alpha_{1}=\cdots=\alpha_{s}=\alpha$, and $\beta_{1}=\cdots=\beta_{s}=\beta$. The assumption made at the beginning of the proof of Theorem 4 implies that the conditions of Proposition 2 are satisfied.

Observations.

1. Since $r(s) \leqslant n$, then $h\left(X_{n}, A_{1}\right)=1$. 
2. Since $r(0)=p$, then $h\left(l_{2}^{n}, A_{s+1}\right)<\beta$.

By Proposition 2 we get

$$
d_{K P}\left(X_{n}, l_{2}^{n}\right) \geqslant(1-\beta)+(s-1)(\alpha-\beta)+(\alpha-\beta)>(s+1)(\alpha-\beta) .
$$

From (4) we get

$$
d_{K P}\left(X_{n}, l_{2}^{n}\right) \geqslant c \cdot \ln \ln n
$$

for some absolute constant $c>0$ if $n$ is large enough. It is clear that (5) contradicts (2).

\section{REFERENCES}

[1] B. Beauzamy, Introduction to Banach spaces and their geometry (North-Holland Publishing Company, Amsterdam, 1982).

[2] J. Bourgain, 'The metrical interpretation of superreflexivity in Banach spaces', Israel $J$. Math. 56 (1986), 222-230.

[3] D. Burago, Y. Burago, and S. Ivanov, A course of metric geometry (American Mathematical Society, Providence, R.I., 2001).

[4] M. Cepedello Boiso, 'Approximation of Lipschitz functions by $\Delta$-convex functions in Banach spaces', Israel J. Math. 106 (1998), 269-284.

[5] R. Deville, G. Godefroy and V. Zizler, Smoothness and renormings in Banach spaces, Pitman Monographs and Surveys in Pure and Applied Mathematics 64, (Longman Scientific \& Technical, Harlow, 1993).

[6] D. van Dulst, Reflexive and superreflexive Banach spaces (Mathematisch Centrum, Amsterdam, 1978).

[7] P. Enflo, 'Banach spaces which can be given an equivalent uniformly convex norm', Israel J. Math. 13 (1972), 281-288.

[8] D.P. Giesy, 'On a convexity condition in normed linear spaces', Trans. Amer. Math. Soc. 125 (1966), 114-146. Additions and corrections to 'On a convexity condition in normed linear spaces', Trans. Amer. Math. Soc. 140 (1969), 511-512.

[9] M. Gromov, 'Groups of polynomial growth and expanding maps', Inst. Hautes Études Sci. Publ. Math. 53 (1981), 53-73.

[10] R.C. James, 'Some self-dual properties of normed linear spaces', in Symposium on infinite dimensional topology, (R.D. Anderson, Editor) (Princeton University Press, Princeton, New Jersey, 1972), pp. 159-175.

[11] R.C. James, 'Super-reflexive spaces with bases', Pacific J. Math. 41 (1972), 409-419.

[12] R.C. James and J.J. Schäffer, 'Super-reflexivity and the girth of spheres', Israel J. Math. 11 (1972), 398-404.

[13] M.I. Kadets, 'Note on the gap between subspaces', (in Russian), Funkts. Anal. Prilozhen. 9 (1975), 73-74. English transl. in Funct. Anal. Appl. 9 (1975), 156-157.

[14] M.I. Kadets, 'The superreflexivity property of a Banach space in terms of the closeness of its finite-dimensional subspaces to Euclidean spaces', Funct. Anal. Appl. 12 (1978), 142-144. 
[15] N.J. Kalton and M.I. Ostrovskii, 'Distances between Banach spaces,', Forum Math. 11 (1999), 17-48.

[16] J. Lindenstrauss and L. Tzafriri, Classical Banach spaces, II, Ergebnisse der Mathematik und ihrer Grenzgebiete 97 (Springer-Verlag, Berlin, 1979).

[17] M.I. Ostrovskii, 'Gap properties and related characteristics of proximity of Banach spaces', (in Russian), Teor. Funktsii Funktsional. Anal. i Prilozhen., 42 (1984), 97-107. English transl. in Amer. Math. Soc. Transl. 136 (1987), 109-119.

[18] M.I. Ostrovskii, 'Banach-Saks properties, injectivity and gaps between subspaces of a Banach space', (in Russian), Teor. Funktsii, Funktsion. Anal. i Prilozhen. 44 (1985), 69-78. English translation.: J. Soviet Math., 48 (1990), no. 3, 299-306.

[19] M.I. Ostrovskii, 'Topologies on the set of all subspaces of a Banach space and related questions of Banach space geometry', Quaestiones Math. 17 (1994), 259-319.

[20] M.I. Ostrovskii, 'Classes of Banach spaces stable and unstable with respect to opening', Quaestiones Math. 19 (1996), 191-210.

[21] M.I. Ostrovskii, 'Paths between Banach spaces', Glasgow Math. J. 44 (2002), 261-273.

[22] G. Pisier, 'Martingales with values in uniformly convex spaces', Israel J. Math. 20 (1975), 326-350.

[23] G. Pisier and Q. Xu, Random series in the real interpolation spaces between the spaces $v_{p}$, Lecture Notes in Math. 1267 (Springer-Verlag, Berlin, 1987), pp. 185-209.

[24] J.J. Schäffer and K. Sundaresan, 'Reflexivity and the girth of spheres', Math. Ann. 184 (1969/1970), 163-168.

[25] J. Wenzel, 'Superreflexivity and $J$-convexity of Banach spaces', Acta Math. Univ. Comenian. (N.S.) 66 (1997), 135-147.

Department of Mathematics

The Catholic University of America

Washington, D.C. 20064

Unitd States of America

e-mail: ostrovskii@cua.edu 